\title{
Mi sueño, vuestro sueño, nuestro sueño
}

\author{
Ana Amália TAvares BAstos BARBosA ${ }^{1}$ \\ aatbb@uol.com.br
}

Recibido: 22/01/13

Aceptado: 26/06/13

\section{RESUMEN}

Este artículo es parte de la tesis doctoral "Tras el cuerpo: una experiencia de arte/educación"

En ella presento, con mayor profundidad, la experiencia que tuve en la organización sin ánimo de lucro Nosso Sonho, entre 2008 y 2011, enseñando arte a un grupo de niño, todos ellos personas con parálisis cerebral y en silla de ruedas, en la etapa de pre-alfabetización. Exploramos diferentes niveles de sensibilidad: proprioceptiva, exteroceptiva, interoceptiva, bajo la luz de una aproximación triangular.

Palabras clave: arte/educación, parálisis cerebral, percepción.

\section{Referencia normalizada}

TAVARES BASTOS BARBOSA; A. (2013). "Mi sueño, vuestro sueño, nuestro sueño". En Arteterapia: Papeles de arteterapia y educación artística para la inclusión social Vol.: 8. Páginas 211-222.

\section{SUMARIO}

Introducción. Los sentidos como órganos de la mente. El inicio del trabajo con los niños. La ampliación del campo cultural de los niños. ¿Cómo surgió la investigación? Observaciones desde la experiencia. Referencias bibliográficas.

\section{My dream, your dream, our dream}

\begin{abstract}
This article is part of my doctoral thesis "BEYOND THE BODY: AN ART/EDUCATION EXPERIENCE".

In it I present, mor fully, the experience I had at a non governamental organization Nosso Sonho, between 2008 and 2011, teaching art to a group of children, all wheel

chairs and cerebral palsy, in their pre-alfabetization. We explored the different levels of sensibylity, proprioceptive, exteroceptive, interceptive under the light of Triangular Approach.
\end{abstract}

Keywords: Art/Education, Cerebral Palsy, Perception.

\section{CONTENTS}

Introduction. Senses as mind's organs. The beginning of the work made with the children. Cultural context's amplification. How did research begin? Observation from experience. References.

\footnotetext{
${ }^{1}$ Associación Nosso Sonho.

http://buscatextual.cnpq.br/buscatextual/visualizacv.do?id=K4277360D7
} 


\section{INTRODUCCIÓN}

Antes de comenzar quiero dejar claro que, a pesar de que mis alumnos tienen parálisis cerebral, o mejor dicho, tienen lesiones cerebrales, o hablando más modernamente, encefalopatía congénita, no soy arteterapeuta, no hago ni pretendo hacer arteterapia, soy educadora de arte y lo que hago es educación artística. Pero creo que todo hacer artístico tiene una función terapéutica.

Con Umbertina Conti Reed (1997pag 305) aprendí que el "cuadro motor secuela de la parálisis cerebral varía en un mismo individuo tanto en cuanto a la naturaleza semiológica como en cuanto a la intensidad". La autora resalta el hecho de que al no ser las lesiones progresivas, ello es un elemento facilitador de la educación.

\section{LOS SENTIDOS COMO ÓRGANOS DE LA MENTE}

En Educación Artística vislumbré la posibilidad de trabajar con lo que Nitrini (1997 pág 12) llama receptores sensoriales y también apoyada en Susanne Langer (1980) que considera a los sentidos como órganos de la mente.

Para Nitrini (1997pág 12 ) existen tres sistemas de sensibilidad: hay tres sistemas de sensibilidad: proprioceptivo, exteroceptivo, interoceptivo, que ella define de la siguiente forma:

"los sistemas exteroceptivos son responsables de la sensibilidad a estímulos externos e incluyen la visión, la audición, la sensibilidad cutánea, el olfato y el paladar. Los sistemas propioceptivos se relacionan con las nociones de posición del cuerpo en el espacio y de los fragmentos del cuerpo en relación a los demás. Los sistemas interoceptivos se responsabilizan de la sensibilidad a estímulos provenientes de vísceras, vasos sanguíneos y otras estructuras internas"

Uno de los objetivos que señalé para mi trabajo fue estimular la sensibilidad propioceptiva y la exteroceptiva. Comencé a trabajar con los niños a partir del cuerpo en el espacio (sensibilidad propioceptiva). Mi propia experiencia comprobaba la importancia del reconocimiento del cuerpo en relación al espacio. ${ }^{2}$

Cuando volví a pintar, uno de mis primeros trabajos fue un autorretrato. Mi médico, el Dr. Ayres Teixeira, que intenta siempre estimularme, me hizo estar de pie, atada en una cama ortostática en vertical frente a un espejo vertical, para que me viese entera. Él me decía:

Mira, tu cuerpo no se mueve, pero tú tienes cuerpo, no eres sólo cabeza.

Es verdad! No puedo moverlo, pero tengo cuerpo. La silla es un instrumento, y no la extensión de mi cuerpo. No fue el primer profesional en hacerme encarar

\footnotetext{
${ }^{2}$ N. De T. Ana Amalia Tavares Bastos Barbosa tiene, al igual que sus alumnos, parálisis cerebral, derivado de un Accidente Vascular Cerebral.
} 
al espejo, pero fue el que acertó con el "clic". Yo tenía que estimular las percepciones sensoriales, corporales y espaciales en los niños. Los niños necesitan tener dominio del propio cuerpo a pesar de ser manipulado por otros. ¡Ese es el principio de la autonomía!

\section{EL INICIO DEL TRABAJO CON LOS NIÑOS}

\section{Autorretrato de Ana Amália 2006}

Esta experiencia en relación con la negación de mi propio cuerpo coincidió con el inicio del trabajo con los niños. Una de las primeras actividades que proyecté para ellos no era ninguna novedad metodológica, pero aprendí con el Abordaje Triangular (Barbosa,1992, Barbosa y Cunha, 2010) que en la experiencia del arte en la educación, no es la novedad lo que tenemos que priorizar sino el contexto, y las necesidades del grupo con el cual se trabaja.

Pedí que me colocaran un rollo de papel kraft en el suelo y propuse a los niños que se tumbaran sobre el papel mientras sus cuidadores dibujaban el contorno de sus cuerpos sobre el papel, después los recortaron y los pintaron y por fin construían escenas en las que las representaciones de sus cuerpos se interrelacionaban unas con otras, dialogaban, jugaban, exploraban el espacio como no lo pueden hacer en la vida real, pues están presos en sillas de ruedas. A través de la representación y de la imaginación, los niños van más allá de las limitaciones que les son impuestas.

Carmela Gross (Barbosa, 1984 pág. 110) ya había trabajado con el contorno de los cuerpos de los alumnos en una actividad en la Pinacoteca del Estado en los años 80,cuando era director Fábio Magalhães. La diferencia era que los niños dibujaban el contorno de los cuerpos unos a otros, y los niños con los que yo trabajo no podían hacerlo. Por tanto para una percepción más aguda del cuerpo en el espacio, enfatizamos el trabajo de creación de diferentes escenas con los dibujos de los cuerpos de los niños. Fue una actividad muy placentera y divertida para ellos y también valiosa para desarrollar la relación de unos con otros y con el espacio

La visita al Instituto Tomie Ohtake para ver y percibir el movimiento de sus cuerpos y de la silla de ruedas, la instrumentalización de sus cuerpos en relación al espacio expositivo y al movimiento de las esculturas de Tomie Ohtake, así como la visita al Parque de la Luz, fueron también guiados por el mismo objetivo: el desarrollo de la sensibilidad propioceptiva para movilizar la mente, ampliar el campo de referencias y estimular el interés cultural.

Recientemente ha tomado notoriedad el estudio del dibujo en relación a la investigación de las relaciones intercerebrales que provoca el acto de dibujar. Los días 28, 29 y 30 de octubre de 2011 tuvo lugar un encuentro en el Departamento de Educación Artística del Teachers College de la Universidad de Columbia, donde estudié y con el cual todavía mantengo comunicación, sobre el dibujo como forma de pensamiento. Están creando en Inglaterra un robot que sea capaz 
de demostrar las funciones cerebrales que moviliza el dibujo. Uno de los trabajos realizados en este encuentro fue tumbar a las personas sobre papel kraft, como yo hice con los niños, y proponer que dibujasen con las dos manos alrededor de sí mismos. El objetivo era otro: mostrar cómo las soluciones gráficas varían de un individuo a otro. La investigación desarrollada con el robot es muy compleja para dar cuenta de esta diversidad. Así, una misma actividad propuesta también varía de acuerdo con la diversidad de objetivos que se quieren conseguir, convirtiéndose entonces en otra actividad, semejante apenas en lo formal.

Otros trabajos como el juguetear con los colores fueron programados para estimular la sensibilidad exteroceptiva, responsable de la captación de estímulos externos, que incluyen la visión, audición, la sensibilidad cutánea, el olfato y el paladar.

Es muy difícil determinar la amplitud de la cognición, de la capacidad de aprender de niños que nacieron con parálisis cerebral . El sistema escolar tiende a rechazarlos o a abandonarlos en el aula. Por lo que aprendí con Jerome Bruner y Elliot Eisner, cuyos libros no cito porque los leí antes del AVC (Accidente Vascular Cerebral)y mi biblioteca fue robada por quien se quedó en mi casa, la mejor actitud pedagógica es alternar actividades muy simples con otras de más alta complejidad, pues la capacidad cognitiva del niño filtra aquello que puede aprender. Yo aseguraba la movilización cognitiva con las actividades muy simples, como colocar colores diferentes con un pincel en el papel, una experiencia que todos podían procesar. Sin embargo, con la ida a los espacios culturales proporcionaba experiencias más complejas, de las que no sabía qué o hasta dónde ellos podrían procesar. La idea era garantizar el mínimo e intentar el máximo, nunca nivelar por abajo con la disculpa de que ellos no pueden entender. En la videografía internacional existen varios documentales de madres luchando para que las autoridades escolares reconozcan que sus hijos con parálisis cerebral tienen capacidad de aprender incluso si no consiguen hablar. El más impresionante de ellos es el vídeo "Las autoridades siempre tienen razón", presentado en el Festival Así Vivimos, de 2009, en el CCBB de São Paulo, y que muestra la lucha de la madre de un niño, que tuvo parálisis cerebral al nacer, para matricularlo en una escuela inglesa de niños normales. Su hijo fue destinado a una escuela de deficientes mentales. Cuando, tras varios años, consiguió la victoria jurídica, la decisión llegaba tarde, el niño acababa de morir.

La web E-How intenta mostrar lo que los profesores deben saber sobre la parálisis cerebral. En esta web, el Dr. Greene, en el artículo" Cerebral Palsy Source: Teaching", dice que el $75 \%$ de los niños con parálisis cerebral tiene inteligencia normal. Menciona relatos de madres, como la madre que es entrevistada en el vídeo inglés antes mencionado, demostrando que hay enorme dificultad para que se acepte que ellos pueden aprender. Mi madre tiene esta experiencia. Incluso personas eruditas la miran con piedad cuando ella afirma que yo tengo la capacidad cognitiva y la memoria perfectas. La próxima pregunta del interlocutor es casi siempre: "¿ella entiende lo que yo digo?....", pronunciado con evidente incredulidad. 
Una recomendación que el Dr. Greene hace es que nunca debemos suponer que un niño con parálisis cerebral no es capaz de hacer o de entender alguna cosa: "Muchas veces los niños os sorprenderán con lo que ellos pueden lograr, a pesar de las discapacidades". Y añade que la mayor parte de los niños con parálisis cerebral, pueden desenvolverse tan bien, o posiblemente mejor, que estudiantes de capacidad media. Como muchas veces no pueden hablar, como la mayoría de mis alumnos, están imposibilitados de demostrar fácilmente sus capacidades cognitivas.

Otros pueden luchar más a causa de déficits mentales, pero se les debe animar para alcanzar las expectativas también ${ }^{3}$.

\section{LA AMPLIACIÓN DEL CAMPO CULTURAL DE LOS NIÑO}

Otro de mis objetivos fue la ampliación del campo cultural de los alumnos, como ya me referí anteriormente. La pedagogía viene dando mucho énfasis al desarrollo cultural como factor propiciador del desarrollo cognitivo, apoyándose en Vygotsky, Paulo Freire y otros culturalistas.

La "privación cultural" viene siendo señalada como una de las causas de las dificultades de aprendizaje. Mis alumnos tienen dificultad de locomoción, sus padres trabajan y tienen poco tiempo para llevarlos a actividades de ocio y espacios culturales. Son padres con una sobrecarga enorme de trabajo doméstico y responsabilidades. Para vencer la "privación cultural" y así ampliar el desarrollo cognitivo no sólo es necesario ponerles en contacto con la cultura, sino conducirlos a pensar sobre ella, a que se conciencien de los valores culturales a los que son expuestos.

Esta es tarea de la escuela, que debe constituirse en un laboratorio de experiencias.

En la web Le Sueur, consultoría y cursos la distancia, encontré un texto sobre el trabajo de Reuven Feuerstein con niños huérfanos de los campos de concentración nazis tras la segunda guerra mundial. Por desgracia, el texto no tiene autor ni bibliografía, pero dice categóricamente: "Feuerstein (1997) rompe con los paradigmas de que la edad y factores orgánicos como la herencia genética, anomalías cromosómicas o emocionales, sean impedimentos para la modificabilidad cognitiva y afectiva, o sean irreversibles y causa central de la falta de aprendizaje". Gómez (2002). ${ }^{4}$

\footnotetext{
${ }^{3}$ Bibliografía consultada en internet en la dirección

http://www.ehow.com/about_5266377_should-teacherscerebral-palsy.html Acceso el día 07/11/2011 a las 14:15

${ }^{4} \mathrm{http}: / /$ www.cursosadistancia.pro.br/teorias_aplicadas/cursos_a_distancia_reuven_feuerstein.htm Acceso día15/12/2011 a las 16:16
} 
En realidad, él ha estado trabajando con mediación cultural o con la consciencia cultural como factor generador de desarrollo cognitivo como investigador y director del International Center for the Enchancement of Learning Potencial en Jerusalén. En la web citada hay un relato atribuido la Feuerstein:

"Durante la Segunda Guerra, viví en campos de concentración y después en prisiones nazis. La guerra acabó y me dediqué a los niños supervivientes del holocausto. Ellos fueron para Israel tras pasar tres o cuatro años en los campos de concentración. Sus padres habían muerto en cámaras de gas. Algunos llegaron a Israel pareciendo esqueletos. Eran totalmente analfabetos a los ocho o nueve años de edad. Yo no podía aceptar que fueran retardados o idiotas. Pasé más de siete años trabajando con esos niños. No conseguían organizar el pensamiento ni sus acciones. Una noche, en Jerusalén, uno de los niños, con ocho años, se acostó a mi lado y entonces comenzamos a leer filosofía juntos. El cambio era posible." ${ }^{5}$

Este es el relato de un caso extremo de privación cultural y de privación de todos los derechos humanos, pero la pedagogía cultural (David Trend, 1992) basada en Paulo Freire y Henry Giroux viene demostrando en la cotidianidad escolar la fuerza de la cultura concientizada para el desarrollo de la cognición.

\section{¿CÓMO SURGIÓ LA INVESTIGACIÓN?}

A mediados de 2007, como parte de mi tratamiento de rehabilitación y de la terapia ocupacional en el Instituto de Medicina Física y de Rehabilitación IMREA, del Hospital de las Clínicas HC, Facultad de Medicina de la Universidad de São Paulo FMUSP, antigua DMR, División de Medicina de Rehabilitación, comencé a impartir talleres de arte. Eran para adultos con alguna deficiencia física. Yo preparaba el aula por escrito, imprimía una copia para cada alumno y la llevaba. El profesor responsable que me acompañaba lo leía junto con los alumnos, ellos cogían el material y trabajaban, al final mirábamos todos los trabajos, yo planteaba algunas cuestiones, mostraba la obra de algún artista y conversábamos.

Yo no imaginaba que sería posible retomar la docencia, a fin de cuentas yo estaba tetrapléjica y muda, la convivencia con los alumnos, fue un gran giro en mi vida. Como no podía comer usaba una sonda naso-enteral que me daba aspecto de enferma. Tras los talleres que di en el IMREA me hice la gastrostomía para poder quitar la sonda naso-enteral y retomar un aspecto más saludable.

En la misma época me inscribí para el doctorado en la ECA/USP.

En el inicio de 2008, Marisa Hirata (terapeutao cupacional que me atiende desde 2004) y Suely Katz(gerente de la ONG Nuestro Sueño) me llamaron para

\footnotetext{
${ }^{5} \mathrm{http} / / /$ www.cursosadistancia.pro.br/teorias_aplicadas/cursos_a_distancia_reuven_feuerstein.ht $\mathrm{m}$
} 
dar clases de arte a un grupo de seis niños, tres niños y tres niñas, en la Asociación Nuestro Sueño. Son niños en pre-alfabetización, en silla de ruedas, todos con déficit visual y de fuerza muscular, tetraparésicos, siendo uno diparético, cinco niños son mudos, los cuales fueron diagnosticados con parálisis cerebral, uno es hidrocefálico, dos sufrieron anoxia de parto, uno de ellos secuelas de bilirrubina, otro secuelas de toxoplasmosis, y un prematuro y gemelo. Me quedé aterrorizada, pero sintiendo un gran desafío. Además, tanto el rango de edad (en la época en que empezamos tenían 7,8 o 9 años, hoy son pre-adolescentes) como la parálisis cerebral me eran totalmente desconocidas. Estudié, respiré hondo y acepté. Mi médico, el Dr. Ayres Teixeira, me dio una bibliografía sobre disfunciones cerebrales. De esa bibliografía lo que más me ayudó fue el libro de Ricardo Nitrini, La Neurología que todo médico debe saber (1997).

Al principio yo daba clase acompañada del arteterapeuta, de la terapeuta ocupacional y de la coordinadora pedagógica. Poco a poco me fueron dejando sola con la profesora. Ya no me asusto más, tengo mis artificios, como la bocina (que yo acciono con la cabeza y que uso para que presten atención).Paralelamente, yo estaba cursando las asignaturas del doctorado y el proyecto fue siendo modificado hasta desembocar en el proyecto que presenté y que recogió el trabajo con los niños.

Se trata de investigación colaborativa, con la participación de especialistas de las diversas áreas de la escuela, especialmente de la profesora de clase.Relacioné la investigación con la Arts Based Research inArt Education, propuesta metodológica lanzada por Elliot Eisner en los años ochenta y revisada en la primera década del dos mil por Ricardo Marín (2010), que la viene practicando con sus orientandos en la Universidad de Granada, España .Se trata de una investigación que sea hace explícita a través de la imagen. Lo que presento aquí es una investigación cuyo relato integra texto e imagen de forma que ambos lenguajes se integren y se completen para la comprensión del lector.

Algunas veces cité textos que escribí en mi blog, en el calor del momento, inmediatamente después de la experiencia, porque de él también vino mucha respuesta positiva y mucho estímulo en los momentos más difíciles de este camino en dirección a un pensamiento más estructurado. El trabajo es investigación cualitativa y su cualidad esencial es la transparencia de la subjetividad del investigador. En el examen de calificación me preguntaron dónde estaba yo, dando a entender que yo debía mostrar más íntegramente mis ideas y circunstancias, no sólo el resultado del trabajo con los alumnos.

A primera vista, mi trabajo de doctorado no tiene relación con mi investigación anterior de máster, que fue sobre una posible interdisciplinaridad entre la enseñanza del inglés y la enseñanza del arte. Mi investigación ahora no es la interdisciplinaridad de contenido, pero adopté la interdisciplinaridad como actitud definida por Ivaní Fazenda (1994):

“... una actitud para conocer más y mejor frente a alternativas, actitud de espera frente ahechos consumados, actitud de reciprocidad que impele al cambio, que impele al diálogo -al diálogo con pares anónimos o uno mismo incluso-, actitud 
de humildad ante la limitación del propio saber, actitud de perplejidad ante la posibilidad de desvelar nuevos saberes, actitud de desafío-desafío ante lo nuevo, desafío en redimensionar lo viejo-, actitud de implicación y compromiso con los proyectos y con las personasen ellos envueltos, actitud, pues, de compromiso en construir siempre de la mejor forma posible, actitud de responsabilidad pero, sobre todo, de alegría, de revelación, de encuentro, y finalmente de vida." (Fazenda, 1994, pp. 31.)

Mi experiencia de volver a enseñar arte tras cinco años de silencio y tetraplejia, como resultado de un AVC de tronco cerebral, fue fundamental para la rehabilitación de la identidad perdida como profesora, que marcó mi vida desde los 15 años de edad, cuando daba clases de arte en las plazas de São Paulo con un grupo de profesoras de la Secretaría de Educación del Estado de São Paulo, en un proyecto de Educación Popular. Aquellas eran clases para niños en los tiempos del modernismo expresionista en arte/educación muy diferentes de las clases que yo planificaba para el taller de artes del IMREA, más informadas culturalmente y más contextualizadas.

\section{OBSERVACIONES DESDE LA EXPERIENCIA}

Como ya dije, en el IMREA yo preparaba la clase y la llevaba escrita para los alumnos, el profesor responsable ya sabía por email de qué se trataba y preparaba el material con antelación. Los alumnos eran adultos con algún déficit motor, secuelas menos severas que las mías y que las de los alumnos con quienes convivo hoy. A continuación, algunas clases en el IMPREA:

OBSERVACIÓN

VAMOS A INTENTAR MIRAR TODO LO QUE NOS RODEA DE FORMA DIFERENTE, COMENCEMOS CON UN OBJETO COMÚN, LA PINZA PARA LA ROPA.

ANTES DE DIBUJAR, COGED UNA PINZA Y SENTID EL PESO, LA TEXTURA Y OBSERVAD COMO FUNCIONA. AHORA, CON UN LÁPIZ, DIBUJAD, DETALLADAMENTE, UNA PINZA DE LA ROPA. TENÉIS 5 MINUTOS.

CAMBIAD LA POSICIÓN DE LA PINZA Y DIBUJAD DE NUEVO, AHORA TENÉIS 1 MINUTO.

CAMBIAD LA POSICIÓN Y DIBUJAD UNA VEZ MÁS. AHORA TENÉIS 30 SEGUNDOS. AHORA DIBUJAD, NUEVAMENTE, DURANTE 5 MINUTOS.

MIRAD LOS DIBUJOS DE LOS OTROS. VAMOS A CONVERSAR UN POCO:

-¿HABÍAIS VISTO ANTES UNA PINZA DE LA ROPA? ROPA?

-¿CUÁNTAS VECES MIRASTEIS ATENTAMENTE UNA PINZA DE LA 
-¿QUÉ OTROS OBJETOS O PERSONAS OS GUSTARÍA MIRAR DE FORMA DIFERENTE?

VARIOS ARTISTAS TRABAJAN A PARTIR DE OBJETOS COMUNES. EL ARTISTA QUE ESTOY MOSTRANDO ES SOLAMENTE UNO.

ANA AMALIA

26/09/07

Presentaba entonces la obra de Claes Oldenburg, que yo introducía así:

TRAS ESTIMULAR LA OBSERVACIÓN DE UN OBJETO DE USO COTIDIANO, UNO DE LOS MÁS BARATOS EN EL MERCADO DE ÚTILES DOMÉSTICOS, BUSCABA IMPULSAR LA IMAGINACIÓN:

ANTES HICISTEIS DIBUJO DE OBSERVACIÓN Y DE MEMORIA DE LAS PINZAS DE ROPA. HAY UN ARTISTA, CLAES OLDENBURG, QUE HIZO UNA ESCULTURA GIGANTE DE UNA PINZA. ¿Y VOSOTROS QUÉ HARÍAIS? USAD VUESTRA IAMGINACIÓN Y CREAD UN DIBUJO EN EL QUE APAREZCAN PINZAS. USAD EL COLOR QUE QUERÁIS Y TODO EL ESPACIO DEL PAPEL.

ANA AMALIA. 31/10/2007

Estos talleres me hicieron volver a creer en el poder organizador del arte para mí y para los adultos con quienes trabajé.

Me sentí tan entusiasmada que propuse un proyecto de dibujo para todos los usuarios de la piscina del IMREA. Según Mauricio Koprowski García, director de la hidroterapia del IMREA, que introdujo en Brasil el método Halliwick:

"Más de mil personas con deficiencia fueron atendidas por el Proyecto Halliwick en la piscina de la unidad Lapa del IMREA desde su implantación hace 4 años. El control de la respiración, del equilibrio y de los movimientos en el agua son los principales objetivos del Método. Las actividades son teniendo en cuenta la habilidad de cada grupo, por lo tanto el programa incluye a todos, independientemente de su deficiencia.

Con apoyo de la Asociación Halliwick Internacional y la Asociación Brasil Halliwick, este es un proyecto pionero en Brasil. Fueron más de 68 mil atenciones e incontables beneficios a los usuarios, exclusivamente personas condeficiencia." (Mauricio Koprowski García, en testimonio a Ana Amalia Barbosa en 2008)

Mauricio Koprowski García es, no sólo muy competente, sino muy cuidadoso con los pacientes. Cuando mi madre buscaba hidroterapia desesperadamente para mí y fui rechazada en todos los lugares, incluso en la AACD, ella contactó con el entonces Secretario de Estado de la Salud, Dr. Barradas, que garantizó que en quince días conseguiría al mejor hidroterapeuta de Brasil para mí. Era Mauricio Koprowski García, que acababa de ser contratado por la Dra. Linamara Batistella para el IMREA, en aquel entonces llamado DMR. Realmente, Mauricio es el mejor profesional que yo podría haber encontrado. Él y Moacir Simplício, mi compañero y amigo de todas las horas, fueron los principales colaboradores del proyecto de dibujo y jurado del proceso de evaluación y selección.

El tema era "Cómo te sientes estando en la piscina". Nosotros, la totalidad de los usuarios de las piscinas del IMREA, no tenemos piscina en casa. Para mí 
estar en el agua es una liberación. Mi cuerpo se hace tan leve que cualquier miembro puede ser manipulado como quiera el terapeuta.

Al contrario, fuera del agua mis manos se cierran a punto de doler mucho, y que alguien pueda doblar mi pierna es muchas veces imposible. Las aplicaciones de toxina butolínica mejoran mucho los dolores, pero la piscina es potenciadora de los efectos positivos de la toxina butolínica. Imaginaba que otros usuarios como yo tenían en la piscina una fuente de placer.

Entregamos a cada usuario papel A4, un lápiz HB2, goma y lápices de cera con colores primarios para llevar a casa, pues pensé también en potenciar que continuaran trabajando autónomamente con el dibujo. Recibimos casi 150 dibujos. Los participantes habían sido avisados de que habría un jurado para selección de los dibujos que irían a formar parte de un gran panel, impreso y en material plástico, que sería exhibido "protempore" en la piscina de la sede de Lapa. La selección de los trabajos fue realizada tomando como base criterios artísticos previamente determinados por el jurado. Los clasificamos en tres grupos; GRUPO 1: Dibujos más expresivos, menos estereotipados y más personales; GRUPO 2: Dibujos que sólo responden nuestra pregunta y GRUPO 3: Dibujo de usuarios que mandaron más de un dibujo, que no responde a nuestra pregunta, o que sólo describen el espacio.

En la inauguración del panel en Lapa estaban presentes los participantes, sus familias, el jurado, Dra. Linamara Batistella, Secretaria de las Personas con Deficiencia del Gobierno del Estado de São Paulo y su equipo y hasta el Secretario de Estado de la Salud, Dr. Barradas, un gran incentivador de los medios de rehabilitación propuestos por la Dra. Linamara. Fue muy estimulante.

Cuando en el inicio de 2008Marisa Hirata, Terapeuta Ocupacional, me propuso llevar la experiencia de arte a una clase de Nuestro Sueño yo estaba muy estimulada por el trabajo que el IMREA me llevó a hacer para mi propia rehabilitación

Marisa justificó su indicación y describió mi actividad de la siguiente manera :

"El trabajo de la terapia ocupacional iniciado con AnaAmália Barbosa en septiembre/2004 se basó en el retorno a las actividades como artista plástica, proponiendo adaptaciones para viabilizar mayor autonomía. La evaluación motora sugirió el uso de la barbilla para realización de pinturas, y junto a ello la base (mesa) necesitó de ajustes para mejor percepción del espacio y control del pincel. Su evolución motora se desarrolló junto a sus perspectivas como artista plástica, haciendo posible una propuesta de reinserción en el mercado de trabajo, no con la intensidad que le permitiría la supervivencia económica, pues esto sería imposible, sino que le permita sentirse útil en la sociedad.

Niños con parálisis cerebral con afectación motora y buen nivel de comprensión se beneficiaron de su trabajo. Ana Amália contó con otros profesionales para la ejecución de su trabajo. La terapia ocupacional tuvo una participación más efectiva en el inicio a través de la indicación y elaboración de las adaptaciones de los materiales utilizados por los alumnos y también en las alteraciones de la propuesta de trabajo para que hubiera mayor participación de los alumnos. 
$29 / 01 / 2008$

HOY POR LA MAÑANA FUÍ A LA ASOCIACIÓN NUESTRO SUEÑO. FUI A CONOCER AL GRUPO DE EDUCACIÓN INFANTIL, SON NIÑOS LINDOS Y CON PARÁLISIS CEREBRAL, UN GRAN DESAFÍO! ME ACORDÉ DE CUANDO COORDINABA A LOS MEDIADORES DE LA EXPOSICIÓN DEL CASTILLO RA-TIM-BUM. SIEMPRE QUE VENÍA UN GRUPO DE NIÑOS DE LA A.A.C.D., YO INTENTABA DESAPARECER. YO NO QUERÍA ASUMIR MI IGNORANCIA. HOY DÍA AÑADO A ESO UN MIEDO GIGANTESCO, DE FRUSTRARLOS. EN LA DMR FORMA PARTE DE MI TRATAMIENTO, Y ES MÁS FACIL CON ADULTOS. (HTTP://AMALIABARBOSA.ZIP.NET)

ESTE ARTÍCULO/PRESENTACIÓN ES, EN REALIDAD, SÓLO UN FRAGMENTO DE MI TESIS DE DOUTORADO "MÁS ALLÁ DEL CUERPO: UNA EXPERIENCIA EN ARTE/EDUCACIÓN", QUE ES UN RELATO FUNDAMENTADO Y DETALLADO DE LA EXPERIENCIA.

COMENZAMOS CON LOS COLORES PRIMARIOS Y SECUNDARIOS, Y CON El EXPRESIONISMO ABSTRACTO. HE AHÍ LOS TRABAJOS FINALES DEL PRIMER SEMESTRE DE 2008.

EN El SEGUNDO SEMESTRE TRABAJAMOS El CUERPO COMO INSTRUMENTO Y SOPORTE.FUERON VARIAS ACTIVIDADES, DOS DESTACARON. UNA EN QUE PINTARON LOS CONTORNOS DE LOS CUERPOS.

Y UNA EN QUE, A PARTIR DE La PERFORMANCE DE YVESKLEIN (QUE ELLOS ASISTIERON VÍA YOUTUBE. http://www.youtube.com/watch? $\mathrm{v}=\mathrm{x}$ 0myzbydipu) $\quad$ ELLOS USARON SUS CUERPOS PARA PINTAR.

EN 2009 COMENZAMOS CON LAS FORMAS, ORGÁNICAS Y GEOMÉTRICAS. HICIMOS UN MÓVIL.

MI INTENCIÓN ERA DE IRNOS A VER El MÓVIL DE CALDER, PERO El LUGAR ERA INACESIBLE.RESOLVÍ, ENTONCES, LLEVARLOS A UNA EXPOSICIÓNDE TOMIE OHTAKE, EN El INSTITUTO TOMIEOHTAKE.

EN 2010 INTRODUJE LO TRIDIMENSIONAL (USAMOS ARCILLA, MASA DE PAN Y CHOCOLATE)Y FUIMOS Al JARDÍN DE LAS ESCULTURAS, EN El PARQUE DE LA LUZ.

AL VOLVER RECIBIMOS LA VISITA DE CAITO (UNODE LOS ARTISTAS CUYA ESCULTURA HABÍAMOSVISTO) QUE HIZO ESCULTURA CON NOSOTROS USANDOMASA DE PAN Y "A LA MANERA DE OSWALD DE ANDRADE" COMEMOS LOSBOLLOS.

YO HABÍA PENSADO EN IR EN TREN AL PARQUE DE La LUZ, PERO NO FUE POSIBLE. EN El SEGUNDOSEMESTRE LO CONSEGUIMOS, Y TRASESTUDIAR El IMPRESIONISMO, FUIMOS EN TREN DESDE BARRA HONDA A LA ESTACIÓN DE LA LUZ. 
AHORA TE CORRESPONDE A TÍ, EXTRAER TUS CONCLUSIONES A PARTIR DE Mi EXPERIENCIA. YO CONTINÚO EN NUESTRO SUEÑO, ES UN PLACERVER A ESOS NIÑOS DESARROLLÁNDOSE.

\section{REFERENCIAS BIBLIOGRÁFICAS}

BARBOSA, Ana Mae (1984). Arte-Educação:conflitos-acertos. SãoPaulo, Editora Max Limonade,1984.

BATEZAT, Maria Lúcia. (2011) Desenho Infantil e Seu Ensino a Crianças Cegas Razões e Método. Curitiba:Editora Insight..

BRAGA, Paula. (2011) "Conceitualismo e Vivência" em BRAGA, Paula.. (Org.). Fios Soltos: A Arte de Hélio Oiticica. São Paulo: Perspectiva. pag 259 a 287.

CASELLA, Erasmo Barbante; AMARO JR,Edison COSTA ,Jaderson Costa da . (2011) "As bases neurobiológicas da aprendizagem da leitura" em ARAUJO,Aloisio (org) Aprendizagem infantil:uma abordagem da neurociência economia e psicologia cognitiva. Rio de Janeiro: Academia Brasileira de Ciências e Fundação Conrado Wessel-pág. 37-69.

CSIKSZENTMIHALYI, Mihaly and ROBINSON,Rick. (1990)The Art of Seeing:an interpretation of the aesthetic encounter.California:A joint publication of the J.Paul Getty Museum and the Getty Center for Education in the Arts.

DEWEY, John. (1934) Arte como Experiência.São Paulo:Ed.Martins Fontes, 2010. A 1 Edição em inglês é de 1934.

FAZENDA, Ivani. (1994) Interdisciplinaridade: História, Teoria e Pesquisa. Campinas S.P., Papirus Editora, 1994.

GARCIA, Mauricio (2008) Koprowski. depoimento a Ana Amália Barbosa.

LANGER, Susane. (1980) Sentimento e Forma.São Paulo: Ed. Perspectiva. 